\title{
E-1 CAM Revision 4
}

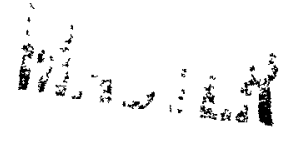

30 Apr11 1970

This report was prepared as an account of work sponsored by the United States Government. Neither the United States nor the United States Energy Research and Development Administration, nor any of their employees, nor any of their contractors, subcontractors, or their employees, makes any waranty, express of implied, or assumes any legal liability or responsiblity for the accuracy, completeness or usefulness of any information, apparatus, product or process disclosed, or represents that its use would not infrunge privately owned rights.
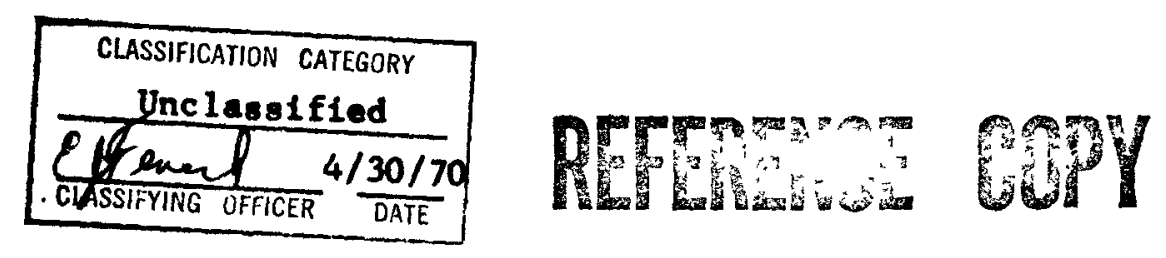

TECHNICAL DOCUMENT CENTER

Nuclear Rocket Operations

DOC. NO.

AGCS $0120-48$ 


\section{DISCLAIMER}

This report was prepared as an account of work sponsored by an agency of the United States Government. Neither the United States Government nor any agency Thereof, nor any of their employees, makes any warranty, express or implied, or assumes any legal liability or responsibility for the accuracy, completeness, or usefulness of any information, apparatus, product, or process disclosed, or represents that its use would not infringe privately owned rights. Reference herein to any specific commercial product, process, or service by trade name, trademark, manufacturer, or otherwise does not necessarily constitute or imply its endorsement, recommendation, or favoring by the United States Government or any agency thereof. The views and opinions of authors expressed herein do not necessarily state or reflect those of the United States Government or any agency thereof. 


\section{DISCLAIMER}

Portions of this document may be illegible in electronic image products. Images are produced from the best available original document. 


\section{TABLE OF CONTENTS}

I. MODEL SUMMARY

$\underline{\text { Revision }}$

Date

A. Feed System

1. Pumps

2. Pump Discharge Line

4

4

$4 / 30 / 70$

$4 / 30 / 70$

B. Reactor and Nozzle

1. Support System Connection to Nozzle Torus

4

$4 / 30 / 70$

2. Skirt/Simulator Tubes

4

$4 / 30 / 70$

3. Nozzle Tubes

4

$4 / 30 / 70$

4. Reflector Inlet Plenum

3

$12 / 22 / 69$

5. Reflector and Peripheral Shield

3

$12 / 22 / 69$

6. Peripheral Shield Exit Plenum

3

$12 / 22 / 69$

7. Turbine/Simulator and Bypass

4

8. Shield Entrance Plenum

3

9. Core and Core Periphery

3

10. Thrust Chamber

$4 / 30 / 70$

11. Section Deleted

12. Support System Inlet and Bypass

4

13. Support System

$12 / 22 / 69$

14. Reactivity

3

$12 / 22 / 69$

15. Kinetics

3

$12 / 22 / 69$

$-$

$4 / 30 / 70$

3

$12 / 22 / 69$

$12 / 22 / 69$

$12 / 22 / 69$

C. Controls, Actuators, and Safety Circuits

1. Nuclear Subsystem Controls

4 
II. DERIVATIONS
A. General
B. Specific

III. MODEL CHECKS

A. Design
1. Steady State
2. Frequency Response
3. Transient Response

4

$4 / 30 / 70$

4

$4 / 30 / 70$

B. Low Power
1. Steady State
2. Frequency Response
3. Transient Response

4

$4 / 30 / 70$

4

$4 / 30 / 70$

IV. NOMENCLATURE

4

$4 / 30 / 70$

APPROVED:

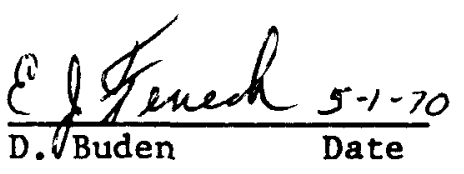

ACKNOWLEDGED:

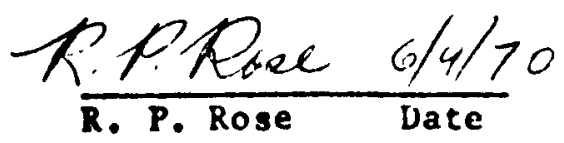




\section{A-1 PUMPS}

$$
\begin{aligned}
& \text { 1. } \mathrm{P}_{3 \mathrm{~A}, \mathrm{~B}}=30, \mathrm{~T}_{1 \mathrm{~B}}=40 \\
& \text { 2. } \mathrm{P}_{4 \mathrm{~A}, \mathrm{~B}}=\mathrm{P}_{3 \mathrm{~A}, \mathrm{~B}}+\mathrm{N}_{\mathrm{A}, \mathrm{B}}^{2} \mathrm{f}_{1}\left(\frac{\mathrm{W}_{\mathrm{PA}, \mathrm{B}}}{\mathrm{N}_{\mathrm{A}, \mathrm{B}}}\right) \\
& \text { 3. } \mathrm{T}_{4 \mathrm{~B}}=\mathrm{T}_{1 \mathrm{~B}}+.015\left(\mathrm{P}_{4 \mathrm{~B}}-\mathrm{P}_{3 \mathrm{~B}}\right) \\
& \text { 4. } \mathrm{M}_{\mathrm{PA}, \mathrm{B}}=\mathrm{N}_{\mathrm{A}, \mathrm{B}}^{2} \mathrm{f}_{2}\left(\frac{\mathrm{W}_{\mathrm{PA}, \mathrm{B}}}{\mathrm{N}_{\mathrm{A}, \mathrm{B}}}\right)
\end{aligned}
$$

5. $\frac{d N_{A, B}}{d t}=114.5\left(M_{T A, B}-M_{P A, B}\right)$ 
TABLE I A-1-1

$\frac{W_{P A, B}}{N A, B}$
$\quad 0 \times 10^{-2}$
.0915
.1112
.1413
.1790
.2165
.2542
.2920
.3300
.3770
.4710

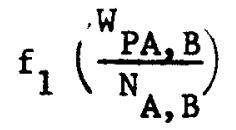

$1.670 \times 10^{-6}$

1.670

1.720

1.700

1.640

1.555

1.440

1.300

1.108

.835

.079
$\mathrm{f}_{2}\left(\frac{\mathrm{W}_{\mathrm{PA}, \mathrm{B}}}{\mathrm{N}_{\mathrm{A}, \mathrm{B}}}\right)$

$1.282 \times 10^{-6}$

1.282

1.103

1.223

1.360

1.505

1.638

1.760

1.855

1.990

2.600 
UNCLASSIFIED

I A-2 PUMP DISCHARGE LINE
Page 1 of 1 Revision: 4 Date: $4 / 30 / 70$

1. $\mathrm{dW}$

$\frac{d W_{P A, B}}{d t}=44.9\left(P_{4 A, B}{ }^{-P_{19}}\right)-.383 W_{P A, B}\left|w_{P A, B}\right|$

$\mathrm{W}_{\mathrm{PA}, \mathrm{B}} \geq 0$

$\therefore \quad \frac{d P_{19}}{d t}=\frac{1810+1.4 P_{19}}{1.489}$

$\left({ }_{P A}+w_{P B}-w_{S S I A}-w_{S S I B}-w_{S S B}-w_{5}\right) \quad w_{P A} \geq 0$

$\frac{\mathrm{dP}_{19}}{\mathrm{dt}}=\frac{1810+1.4 \mathrm{P}_{19}}{1.002}$

$\left(w_{P A}+w_{P B}-w_{S S I A}-w_{S S I B}-w_{S S B}-w_{5} ; \quad w_{P A}=0\right.$

3. $\mathrm{T}_{19}=\mathrm{T}_{4 \mathrm{~B}}$ 
I B-1 SUPPORT SYSTEM CONNECTION TO NOZZLE TORUS

$$
\begin{aligned}
\therefore \quad \frac{d W_{5}}{d t} & =19.14\left(P_{19}-P_{5}\right)-.078 W_{5}\left|W_{5}\right| \\
\therefore \quad \frac{45}{12} & =\frac{1810+1.4 P_{5}}{4.25}\left(W_{5}-W_{N C}\right) \\
\therefore \quad T_{5} & =T_{4 B}
\end{aligned}
$$


Page 1 of 1

UNCLASS IF IED

\section{B-2 SKIRT/SIMULATOR TUBES}

$$
\begin{aligned}
& \therefore \frac{d T_{S K C}}{d t}=.073\left[\left..700 i \mathrm{w}_{N C}\right|^{.8}\left(\psi_{S K}-T_{S K C}\right)-1.40\left|\mathrm{w}_{N C}\right|^{.8}\left(T_{S K C}-\rho_{J}\right)-4.00 \mathrm{~h}_{N C}\left(T_{S K C}{ }^{-T_{5}}\right)\right] \\
& \text { 2. } \frac{d H_{S K}}{d t}=.00825\left[.218\left|\mathrm{w}_{\mathrm{N}}\right|^{.8}\left(\mathrm{~T}_{15}-\varphi_{\mathrm{SK}}\right)-.700\left|\mathrm{w}_{\mathrm{NC}}\right|^{.8}\left(\varphi_{\mathrm{SK}}-\mathrm{T}_{\mathrm{SKC}}\right)\right] \\
& \therefore{ }^{\prime}{ }_{S K}=7.94 H_{S K}+200
\end{aligned}
$$




\section{B-3 NOZZLE TUBES}

1. $\frac{\mathrm{dT}}{\mathrm{NC}}=1.61\left[.443\left|\mathrm{~W}_{\mathrm{NC}}\right|^{\cdot 8}\left(\varphi_{\mathrm{N}}-\mathrm{T}_{\mathrm{NC}}\right)-.38\left|\mathrm{~W}_{\mathrm{NC}}\right|^{.8}\left(\mathrm{~T}_{\mathrm{SKC}}-\varphi_{\mathrm{J}}\right)-4.00 \mathrm{~W}_{\mathrm{NC}}\left(\mathrm{T}_{\mathrm{NC}}-\mathrm{T}_{\mathrm{SKC}}\right)\right]$

2. $\frac{\mathrm{dH}}{\mathrm{dt}}=.0514\left[.133\left|\mathrm{H}_{\mathrm{N}}\right|^{.8}\left(\mathrm{~T}_{15}-\varphi_{\mathrm{N}}\right)-.443\left|\mathrm{~W}_{\mathrm{NC}}\right|^{\cdot 8}\left({ }_{\mathrm{N}}-\mathrm{T}_{\mathrm{NC}}\right)\right]$

3. $\frac{\mathrm{dH}_{\mathrm{J}}}{\mathrm{dt}}=.00282\left|\mathrm{~W}_{\mathrm{NC}}\right|^{\cdot 8}\left(\mathrm{~T}_{\mathrm{SKC}}-\varphi_{\mathrm{J}}\right)$

4. $\varphi_{\mathrm{N}}=8.93 \mathrm{H}_{\mathrm{N}}+100$

5. $\varphi_{\mathrm{J}}=16 \mathrm{H}_{\mathrm{J}}$

6. $\frac{d W_{N C}}{d t}=128.5\left(P_{5}-P_{7}-\frac{.0361}{R_{7}} W_{N C}\left|W_{N C}\right|\right)$ 
Page 1 of 3

UNCLASS IFIED

Revision: 4

Date: $4 / 30 / 70$

\section{B-4 REFLECTOR INLET PLENUM}

1. $\frac{\mathrm{dR}_{7}}{\mathrm{dt}}=.352\left(\mathrm{~W}_{\mathrm{NC}}-\mathrm{W}_{\mathrm{R}}\right)$

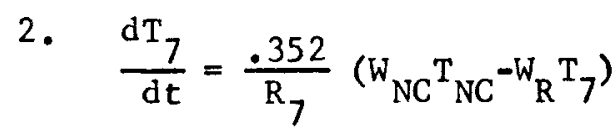

3. $P_{7}=T_{7} f_{1}\left(R_{7}\right)-f_{2}\left(R_{7}\right)$

4. $\mathrm{T}_{7 \mathrm{~min}}=52 .+.043 \mathrm{P}_{7}$

$P_{7}<210$ psia 
UNCLASSIFIED

Page 2 of 3 Revision: 4 Date: $4 / 30 / 70$

\section{B-4 REFLECTOR INLET PLENUM}

TABLE I B-4-1

${ }_{7}\left(1 \mathrm{~b} / \mathrm{ft}^{3}\right)$
0.0000
0.2516
0.5032
1.040
1.384
1.824
2.390
2.768
3.500
3.744

$$
\begin{gathered}
f_{2}\left(R_{7}\right)(p s i a) \\
0.0 \\
15.63 \\
60.06 \\
224.2 \\
410.7 \\
674.7 \\
1089.0 \\
1420.0 \\
2160.0 \\
2371.0
\end{gathered}
$$


UNCLASSIFIED

Page 3 of 3

Revision: 4

Date: $4 / 30 / 70$

\section{B-4 REFLECTOR INLET PLENUM}

TABLE I B-4-2

$\begin{array}{lc}R_{7}\left(1 \mathrm{~b} / \mathrm{ft}^{3}\right) & \mathrm{f}_{1}\left(\mathrm{R}_{7}\right)\left(\mathrm{psia}{ }^{\circ} \mathrm{R}\right) \\ 0.0000 & 0.000 \\ 0.2516 & 1.426 \\ 0.6290 & 3.881 \\ 0.8806 & 5.733 \\ 1.132 & 7.787 \\ 1.635 & 12.53 \\ 2.020 & 16.62 \\ 2.520 & 23.20 \\ 3.019 & 31.87 \\ 3.271 & 37.15 \\ 3.744 & 49.82\end{array}$




\section{B-5 REFLECTOR AND PERIPHERAL SHIELD}

$$
\begin{aligned}
& \therefore \quad \frac{i}{d r} H_{r}=2.19 \times 10^{-4}\left(4.310\left|\mathrm{w}_{\mathrm{r}}\right|\left(\mathrm{T}_{7}-\varphi_{\mathrm{r}}\right)+208.6 \mathrm{~S}_{\mathrm{NO}}\right) \\
& \therefore \quad \vartheta_{r}=\mathrm{f}\left(\mathrm{H}_{\mathrm{r}}\right) \\
& \therefore \quad \ddot{r}_{g}=1.16 \varphi_{\mathrm{r}}-(1.16-1) \mathrm{T}_{7} \\
& \therefore \quad w_{\mathrm{r}}=10.2\left(\mathrm{R}_{10}\left|\mathrm{P}_{7}-\mathrm{P}_{10}\right|\right)^{.5}\left[\frac{\left(\mathrm{P}_{7}-\mathrm{P}_{10}\right)}{\left|\mathrm{P}_{7}-\mathrm{P}_{10}\right|}\right]
\end{aligned}
$$


I B-5 REFLECTOR AND PERIPHERAL SHIELD

TABLE 1 B-5-1

$$
\varphi_{r} \text { vs } H_{r}
$$

$\begin{array}{cc}H_{r}(B T U / 1 b) & \varphi_{r}\left({ }^{\circ} R\right) \\ 0 & 0 \\ 5.84 & 185 \\ 11.15 & 235 \\ 16.86 & 275 \\ 22.95 & 310 \\ 27.93 & 335 \\ 33.43 & 360 \\ 39.35 & 385 \\ 44.35 & 405 \\ 49.57 & 425 \\ 55.00 & 445 \\ 60.68 & 465 \\ 65.08 & 480 \\ 106.06 & 605\end{array}$


UNCLASSIFIED

I B-6 PERIPHERAL SHIELD EXIT PLENUM

$$
\begin{aligned}
& \therefore \frac{\mathrm{d}}{\mathrm{d} t} \mathrm{R}_{10}=.0848\left(\mathrm{~W}_{\mathrm{r}}+\mathrm{W}_{s s}+\mathrm{W}_{\text {ssb }}-\mathrm{W}_{11}\right) \\
& 2 . \frac{\mathrm{d}}{\mathrm{d} t} \mathrm{~T}_{10}=.1841\left(\mathrm{~W}_{\mathrm{r}} \mathrm{T}_{9}+\mathrm{W}_{s s} \mathrm{~T}_{22}+\mathrm{W}_{s s b} \mathrm{~T}_{19}-\mathrm{W}_{11} \mathrm{~T}_{10}\right) \\
& \therefore \quad \mathrm{P}_{10}=5.32 \mathrm{R}_{10} \mathrm{~T}_{10}
\end{aligned}
$$

Page 1 of 1 Revision: 4 Date: $4 / 30 / 70$ 


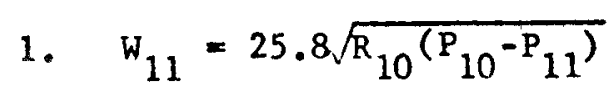

2. $\frac{\mathrm{dR}_{11}}{\mathrm{dt}}=.198\left(\mathrm{~W}_{11}-\mathrm{w}_{\mathrm{BCVA}}-\mathrm{w}_{\mathrm{BCVB}}-\mathrm{W}_{\mathrm{TA}}-\mathrm{W}_{\mathrm{TB}}\right)$

3. $\mathrm{T}_{11}=\mathrm{T}_{10}$

4. $\mathrm{P}_{11}=5.32 \mathrm{R}_{11} \mathrm{~T}_{11}$

5. $\mathrm{W}_{\mathrm{TA}, \mathrm{B}}=6.94 \cdot \frac{\mathrm{P}_{11}}{\sqrt{\mathrm{T}_{11}}} \mathrm{~F}\left(\frac{\mathrm{P}_{11}}{\mathrm{P}_{12 \mathrm{~A}, \mathrm{~B}}}\right)$

6. $\mathrm{E}_{11}=3.145 \mathrm{~T}_{11}-95.9$

$\mathrm{T}_{11} \leq 221$

$=3.831 \mathrm{~T}_{11}-247.5$

$\mathrm{T}_{11}>221$

7. $S O P Y=f\left(E_{11}, P_{11}\right)$

8. $\Delta E_{S A, B}=E_{11}-f\left(S O P Y, P_{12 A, B}\right)$

9. $\mathrm{C}_{\mathrm{OA}, \mathrm{B}}=224 \sqrt{\Delta E_{\mathrm{SA}, \mathrm{B}}}$

10. $\left(\frac{\mathrm{U}}{\mathrm{C}_{\mathrm{O}}}\right)_{\mathrm{A}, \mathrm{B}}=.0305 \frac{\mathrm{N}_{\mathrm{A}, \mathrm{B}}}{\mathrm{C}_{\mathrm{OA}, \mathrm{B}}}$

?. $\mathrm{ET}_{A, B}=\mathrm{E}\left(\frac{\mathrm{U}}{\mathrm{C}_{\mathrm{O}}}\right)_{\mathrm{A}, \mathrm{B}}$

12. $\mathrm{M}_{\mathrm{TA}, \mathrm{B}}=7430 \frac{\mathrm{W}_{\mathrm{TA}, \mathrm{B}}}{\mathrm{N}_{\mathrm{A}, \mathrm{B}}} \mathrm{ET}_{\mathrm{A}, \mathrm{B}} * \Delta \mathrm{E}_{\mathrm{SA}, \mathrm{B}}$

13. $E_{12 A, B}=E_{11}-E T_{A, B} * \Delta E_{S A, B}$ 
UNCLASSIFIED

I B-7 TURBINE/SIMULATOR AND BYPASS
Page 2 of 6 Revision: 4

Date: $4 / 30 / 70$

14. $\frac{\mathrm{dR}_{12 \mathrm{~A}, \mathrm{~B}}}{\mathrm{dt}}=1.00\left(\mathrm{~W}_{\mathrm{TA}, \mathrm{B}}-\mathrm{W}_{\mathrm{SCVA}, \mathrm{B}}\right)$

15. $\mathrm{T}_{12 \mathrm{~A}, \mathrm{~B}}=.318 \mathrm{E}_{12 \mathrm{~A}, \mathrm{~B}}+30.5$

$\mathrm{E}_{12 \mathrm{~A}, \mathrm{~B}} \leq 600$

$=.261 \mathrm{E}_{12 \mathrm{~A}, \mathrm{~B}}+64.6$

$E_{12 A, B}>600$

16. $\mathrm{P}_{12 \mathrm{~A}, \mathrm{~B}}=5.32 \mathrm{R}_{12 \mathrm{~A}, \mathrm{~B}} \mathrm{~T}_{12 \mathrm{~A}, \mathrm{~B}}$

17. $\mathrm{W}_{\mathrm{SCVA}, \mathrm{B}}=.166 \theta_{\mathrm{SCVA}, \mathrm{B}} \sqrt{\mathrm{R}_{12 \mathrm{~A}, \mathrm{~B}}\left(\mathrm{P}_{12 \mathrm{~A}, \mathrm{~B}}-\mathrm{P}_{13}\right)}$

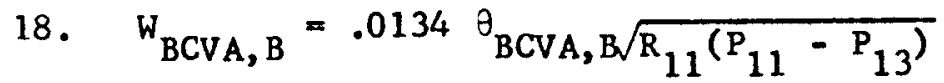

19. $\frac{\mathrm{d} \mathrm{T}_{13}}{\mathrm{dt}}=\frac{.148}{\mathrm{R}_{13}}\left[\mathrm{~W}_{\text {SCVA }} \mathrm{T}_{12 \mathrm{~A}}+\mathrm{W}_{\text {SCVB }} \mathrm{T}_{12 \mathrm{~B}}+\left(\mathrm{W}_{B C V A}+\mathrm{W}_{B C V B}\right) \mathrm{T}_{11}-\mathrm{W}_{14} \mathrm{~T}_{13}\right]$

20. $\frac{d R_{13}}{d t}=.148\left(w_{S C V A}+w_{S C V B}+w_{B C V A}+w_{B C V B}-w_{14}\right)$

21. $\mathrm{P}_{13}=5.32 \mathrm{R}_{13^{\mathrm{T}}} \mathrm{T}_{13}$

22. $W_{14}=26.4 \sqrt{R_{13}\left(P_{13}-P_{14}\right)}$ 
TABLE I B-7-1

FLOW PARAMETER

$\mathrm{P}_{11}$
$\mathrm{P}_{12 \mathrm{~A}, \mathrm{~B}}$
1.0
1.1
1.2
1.4
1.6
1.8
2.0
2.2
2.4
2.6

$$
\begin{gathered}
f\left(\frac{P_{11}}{P_{12 A, B}}\right) \\
0 \\
.0460 \\
.0655 \\
.0840 \\
.0952 \\
.1028 \\
.1070 \\
.1100 \\
.1115 \\
.1127
\end{gathered}
$$


UNCLASSIFIED

Page 4 of 6 Revision: 4 Date: $4 / 30 / 70$

\section{TABLE I B-7-2}

TURBINE EFFICIENCY

$\left(\frac{\mathrm{U}}{\mathrm{C}_{\mathrm{O}}}\right)_{\mathrm{A}, \mathrm{B}}$
0
.2
.3
.4
.5
.6
.7
.8
.9
1.2

$$
\begin{gathered}
f\left(\frac{U}{C_{0}}\right)_{A, B} \\
.770 \\
.870 \\
.907 \\
.910 \\
.875 \\
.807 \\
.715 \\
.615 \\
.508 \\
.160
\end{gathered}
$$


TABLE I B-7-3

TURBINE ENTROPY

SOPY $=f\left(E_{11}, P_{12}\right)$

$\begin{array}{rrrrrrrrr}\underline{P} & \underline{E}=300 & 400 & \underline{500} & \underline{600} & \underline{700} & \underline{800} & \underline{900} & \underline{1000} \\ 20 & 10.431 & 10.690 & 11.267 & 11.758 & 12.189 & 12.577 & 12.929 & 13.252 \\ 50 & 9.517 & 9.789 & 10.364 & 10.854 & 11.285 & 11.673 & 12.024 & 12.347 \\ 100 & 8.816 & 9.109 & 9.682 & 10.170 & 10.601 & 10.988 & 11.339 & 11.662 \\ 200 & 8.097 & 8.431 & 8.999 & 9.485 & 9.914 & 10.300 & 10.652 & 10.974 \\ 300 & 7.662 & 8.035 & 8.598 & 9.083 & 9.511 & 9.896 & 10.247 & 10.570 \\ 400 & 7.344 & 7.755 & 8.314 & 8.796 & 9.223 & 9.608 & 9.959 & 10.281 \\ 500 & 7.090 & 7.537 & 8.093 & 8.573 & 8.999 & 9.383 & 9.734 & 10.056 \\ 600 & 6.878 & 7.360 & 7.912 & 8.393 & 8.815 & 9.199 & 9.549 & 9.872 \\ w 0 & 6.695 & 7.209 & 7.758 & 8.235 & 8.659 & 9.042 & 9.392 & 9.715 \\ 100 & 6.534 & 7.078 & 7.624 & 8.099 & 8.523 & 8.906 & 9.256 & 9.578 \\ 4,0 & 6.391 & 6.962 & 7.505 & 7.979 & 8.402 & 8.785 & 9.134 & 9.457 \\ 106 & 6.262 & 6.857 & 7.398 & 7.871 & 8.293 & 8.676 & 9.025 & 9.347 \\ 1100 & 6.146 & 6.762 & 7.301 & 7.773 & 8.195 & 8.577 & 8.926 & 9.248 \\ 1200 & 6.043 & 6.675 & 7.212 & 7.683 & 8.104 & 8.486 & 8.835 & 9.157 \\ 1400 & 5.892 & 6.518 & 7.052 & 7.521 & 7.942 & 8.323 & 8.672 & 8.995\end{array}$


UNCLASSIFIED

TABLE I B-7 -4

ISENTROPIC EXIT ENTHALPY

$$
f\left(S_{0 P Y}, P_{12 A, B}\right)
$$

Page 6 of 6 Revision: 4 Date: $4 / 30 / 70$

\begin{tabular}{|c|c|c|c|c|c|c|c|c|c|c|c|c|c|}
\hline SOPY & $\underline{P}_{12 A, B}$ & 20 & 50 & 100 & 200 & 300 & 400 & 500 & 600 & $\underline{700}$ & 800 & $\underline{900}$ & 1000 \\
\hline 8.0 & & 0 & 0 . & 0 . & 0. & 386 & 437 & 479. & 515. & 545. & 575. & 605. & 626. \\
\hline 8.2 & & 0 & 0. & 0 . & 322 . & 423. & 476. & 520. & 556. & 593. & 620. & 642. & 674. \\
\hline 8.4 & & 0 & 0. & 0 . & 388 & 459. & 515. & 558. & 600. & 633. & 666. & 700 & 725 . \\
\hline 8.6 & & 0 & 0. & 0 . & 421. & 501. & 555. & 606. & 645. & 683. & 716. & 747. & 778. \\
\hline 8.8 & & 0 & 0. & 295 & 455. & 534. & 600 & 648. & 696. & 733. & 767. & 802 . & 830 . \\
\hline 9.0 & & 0 & 0 . & 347 & 500 & 579. & 643 & 701. & 744. & 785. & 823. & 857. & 891. \\
\hline 9.2 & & 0. & 0 . & 406 & 531. & 625 & 695. & 748. & 801 . & 840 . & 881. & 918. & 951. \\
\hline 9.4 & & 0. & 0. & 433. & 578. & 670 & 741. & 805 . & 854. & 902. & 940. & 980. & 1015 \\
\hline 9.6 & & 0 . & 325. & 475 & 620 & 717 & 798 & 855. & 910. & 958. & 1005 & 1039 & 1072 \\
\hline 9.8 & & 0. & 400. & 520 & 664 & 769 & 848 & 916. & 975. & 1024 & 1065 & 1106 & 1148 \\
\hline
\end{tabular}

Extrapolation should be provided 
Page 1 of 1

UNCLASSIF IED

Revision: 4

Date: $4 / 30 / 70$

\section{B-8 SHIELD ENTRANCE PLENUM}

1. $\frac{\mathrm{dR}}{14} \mathrm{dt}=.1844\left(\mathrm{~W}_{14}-\mathrm{W}_{\mathrm{C}}\right)$

2. $\frac{\mathrm{dT}_{14}}{\mathrm{dt}}=.561\left(\mathrm{~W}_{14} \mathrm{~T}_{13}-\mathrm{W}_{\mathrm{C}^{\mathrm{T}} 14}\right)$

3. $\mathrm{P}_{14}=5.32 \mathrm{R} 14^{\mathrm{T}} 14$ 


\section{B-9 CORE AND CORE PERIPHERY}

$$
\begin{aligned}
& \text { 1. } \frac{\mathrm{d}}{\mathrm{dt}} \mathrm{H}_{\mathrm{cl}}=0.0009772\left(3.753\left|\mathrm{w}_{\mathrm{c}}\right|\left(\mathrm{T}_{14}-\varphi_{\mathrm{cl}}\right)+4432 . \mathrm{S}\right) \\
& \text { 2. } \varphi_{\mathrm{cl}}=\mathrm{f}\left(\mathrm{H}_{\mathrm{cl}}\right) \\
& \text { 3. } \mathrm{T}_{\mathrm{c} 1 \mathrm{e}}=0.98 \varphi_{\mathrm{c} 1}-(.98-1) \mathrm{T}_{14} \\
& \text { 4. } \frac{\mathrm{d}}{\mathrm{dt}} \mathrm{H}_{\mathrm{c} 2}=0.0009772\left(3 . 7 1 1 | \mathrm { w } _ { \mathrm { c } } | \left(\mathrm{~T}_{\mathrm{cl}} \mathrm{e}^{\left.\left.-\varphi_{\mathrm{c} 2}\right)+6564 . \mathrm{S}\right)}\right.\right.
\end{aligned}
$$$$
\text { 5. } \varphi_{c 2}=f\left(H_{c 2}\right)
$$$$
\text { 6. } \mathrm{T}_{\mathrm{c} 2 \mathrm{e}}=0.9690_{c 2}-(.969-1) \mathrm{T}_{\mathrm{cle}}
$$$$
\text { 7. } \frac{\mathrm{d}}{\mathrm{dt}} \mathrm{H}_{\mathrm{c} 3}=0.0009772\left(3.659\left|\mathrm{w}_{\mathrm{c}}\right|\left(\mathrm{T}_{\mathrm{c} 2 \mathrm{e}}-\varphi_{\mathrm{c} 3}\right)+3486 . \mathrm{S}\right)
$$

8. $\varphi_{\mathrm{c} 3}=\mathrm{f}\left(\mathrm{H}_{\mathrm{c} 3}\right)$

9. $\mathrm{T}_{15}=\left(.9554 \varphi_{c 3}-(.9554-1) \mathrm{T}_{\mathrm{c} 2 \mathrm{e}}\right) .9900$

10. $\varphi_{c}=.125\left(3 \varphi_{c 1}+2 \varphi_{c 2}+3 \varphi_{c 3}\right)$

11. $R_{c}=0.138 R_{14}+.841 R_{15}$

12. $\mathrm{W}_{c}=\left[\frac{\left|.9394 \mathrm{P}_{14}-\mathrm{P}_{15}\right|}{\frac{.002604}{\mathrm{R}_{14}}+\frac{.001198}{\mathrm{R}_{c}}}\right]^{0.5} \cdot\left[\frac{\left(0.9394 \mathrm{P}_{14}-\mathrm{P}_{15}\right)}{\left|0.9394 \mathrm{P}_{14}-\mathrm{P}_{15}\right|}\right]$ 
I B-9 CORE AND CORE PERIPHERY

TABLE 1 B-9-1

$\varphi_{\mathrm{cn}}$ v8 $\mathrm{H}_{\mathrm{cn}} \mathrm{n}=1,2,3$

$H_{c n}(B T U / 1 b)$

0

200

400

600

800

1000

1200

1400

1600

1800

2000

2200

2400

$$
\psi_{c n}\left({ }^{O} R\right)
$$

0

1110

1630

2085

2530

2950

3350

3760

4170

4580

4990

5400

5810 


\section{B-10 THRUST CHAMBER}

1. $\frac{\mathrm{dR}_{15}}{\mathrm{~d} t}=.0541\left(\mathrm{~W}_{\mathrm{C}}-\mathrm{W}_{\mathrm{N}}\right)$

2. $\mathrm{P}_{15}=5.32 \mathrm{R}_{15} \mathrm{~T}_{15}$

3. $\mathrm{W}_{\mathrm{N}}=13.01 \frac{\mathrm{P}_{15}}{\sqrt{\Gamma_{15}}}$

4. $\frac{\mathrm{dT}_{15 \mathrm{M}}}{\mathrm{dt}}=\frac{\mathrm{W}_{\mathrm{N}}^{.5}}{2.85}\left(\mathrm{~T}_{15} \mathrm{~T}_{15 \mathrm{M}}\right)$ 
I B-12 SUPPORT SYSTEM INLET AND BYPASS

1. $\ni_{\text {SSVA, B }}=$ Independent Variable

2. $\mathrm{W}_{\text {SSIA, } B}=.0362 \theta_{\text {SSVA, } B}\left|\mathrm{P}_{19}-\mathrm{P}_{20}\right| \cdot 5\left[\frac{\left(\mathrm{P}_{19}-\mathrm{P}_{20}\right)}{\left|\mathrm{P}_{19}-\mathrm{P}_{20}\right|}\right]$

3. $\left.\mathrm{W}_{\mathrm{SSB}}=.1657 \mid \mathrm{P}_{19}-\mathrm{P}_{10} \cdot 5 \cdot \frac{-\left(\mathrm{P}_{19}-\mathrm{P}_{10}\right)}{\left|\mathrm{P}_{19}-\mathrm{P}_{10}\right|}\right]$

4. $\frac{\mathrm{d}}{\mathrm{dt}} \mathrm{P}_{20}=400\left(\mathrm{~W}_{\mathrm{SSIA}}+\mathrm{W}_{\mathrm{SSIB}}-\mathrm{W}_{\mathrm{SS}}\right)$ 


\section{B-13 SUPPORT SYSTEM}

$$
\begin{aligned}
& \text { 1. } \mathrm{T}_{20}=\mathrm{T}_{19} \\
& \text { 2. } \mathrm{T}_{881}=\mathrm{T}_{20}+\left[\frac{0.07711}{1+0.4165 \mid \mathrm{W}_{88} \mathrm{~T}}\right]\left(\mathrm{T}_{\mathrm{s84}}-\mathrm{T}_{\mathrm{s81} 1}\right) \\
& \text { 3. } \mathrm{T}_{\text {ssle }}=2 \mathrm{~T}_{\text {ss1 }}-\mathrm{T}_{20} \\
& \text { 4. } T_{s s 2}=T_{s s 1 e}+0.9136\left(\varphi_{t 2 j}-T_{s s 2}\right) \\
& \text { 5. } \mathrm{T}_{882 \mathrm{e}}=1.5 \mathrm{~T}_{882}-0.5 \mathrm{~T}_{\text {s81e }} \\
& \text { 6. } T_{s 83}=T_{s 82 e}+3.01\left(\varphi_{t 20}-T_{s 83}\right)+3.46\left(\varphi_{t 3 j}-T_{s s 3}\right) \\
& \text { 7. } \mathrm{T}_{883 \mathrm{e}}=1.2 \mathrm{~T}_{\mathrm{ss} 3}-0.2 \mathrm{~T}_{\mathrm{ss} 2 \mathrm{e}} \\
& \text { 8. } \mathrm{T}_{884}=\mathrm{T}_{883 e}+\left[\frac{0.1089}{1+0.4165\left|\mathrm{~W}_{88}\right|}\right]\left(\mathrm{T}_{s 81}-\mathrm{T}_{\text {ss4 }}\right) \\
& \text { 9. } \mathrm{T}_{22}=2 \mathrm{~T}_{\text {ss4 }}-\mathrm{T}_{\text {ss } 3 e} \\
& \text { 10. } \varphi_{t 2 j}=\varphi_{t 2}+0.1907\left|w_{s s}\right|\left(T_{s s 2}-\varphi_{t 2 j}\right) \\
& \text { 11. } \varphi_{t 20}=\varphi_{t 2}+0.5008 \mid W_{s s} i\left(T_{s 83}-\varphi_{t 2 o}\right) \\
& \text { 12. } \varphi_{t 3 j}=\varphi_{t 3}+0.02082\left|w_{s 8}\right|\left(T_{s s 3}-\varphi_{t 3 j}\right) \\
& \text { 13. } \frac{\mathrm{d}}{\mathrm{dt}} \varphi_{\mathrm{t} 2}=3.416\left(\varphi_{t 20}-\varphi_{\mathrm{t} 2}\right)-2.968\left(\varphi_{t 2}-\varphi_{t 2 j}\right)+2.255 \mathrm{~S}_{\mathrm{NQ}} \\
& \text { 14. } \frac{\mathrm{d}}{\mathrm{dt}} \varphi_{t 3}=0.5645\left(\varphi_{\mathrm{p}}-\varphi_{\mathrm{t} 3}\right)-60.00\left(\varphi_{t 3}-\psi_{\mathrm{t} 3 \mathrm{j}}\right)+2.123 \mathrm{~S}_{\mathrm{NQ}} \\
& \text { 15. } \frac{d}{d t} \varphi_{p}=0.195\left(\varphi_{s g}-\varphi_{p}\right)-0.1474\left(\varphi_{p}-\varphi_{t 3}\right)+1.015 S_{N Q}
\end{aligned}
$$




\section{B-13 SUPPORT SYSTEM}

16. $\frac{\mathrm{d}}{\mathrm{dt}} \varphi_{\mathrm{sg}}=0.5476\left(\varphi_{\mathrm{c} 2}-\varphi_{\mathrm{sg}}\right)-0.07476\left(\varphi_{\mathrm{sg}}-\varphi_{\mathrm{p}}\right)+0.92 \mathrm{~S}_{\mathrm{NQ}}$

17. $\mathrm{R}_{882}=.0813+.02515 \times 10^{-3} \mathrm{P}_{20}+1.16 \mathrm{R}_{\mathrm{s8} 2 \mathrm{e}}$

18. $P_{\text {ss } 2 e}=P_{20}-.2296\left(P_{20}-P_{10}\right)$

19. $R_{s s 2 e}=P_{s s 2} e^{/ 5.32 T} s s 2 e$

20. $R_{s s}=0.673 R_{s s 2}+(1-0.673)(0.693) R_{8 s 2 e}$

$21 \mathrm{~W}_{s 8}=1.398\left(\mathrm{R}_{882 \mathrm{e}}\left|\mathrm{P}_{20}-1.06 \mathrm{P}_{10}\right|\right)^{.5}\left[\frac{\left(\mathrm{P}_{20}-1.06 \mathrm{P}_{10}\right)}{\mid \mathrm{P}_{20}-1.06 \mathrm{P}_{10}}\right\rfloor$ 


\section{B-14 REACTIVITY}

$$
\begin{aligned}
& \text { 1. } \mathrm{Dk}_{\mathrm{f}}=11.52 \mathrm{R}_{\mathrm{c}}+2.27 \mathrm{R}_{\mathrm{ss}}-8.86 \times 10^{-4}\left(\varphi_{c}-520\right)+4.6 \times 10^{-4}\left(\varphi_{\mathrm{r}}-520\right) \\
& \text { 2. } \mathrm{DK}_{\mathrm{d}}=6.0 \mathrm{sin}^{2}\left(\frac{{ }^{\theta} \mathrm{D}_{\mathrm{M}}}{2}\right)-1.65 \\
& \text { 3. } \mathrm{Dk}_{\mathrm{t}}=\mathrm{Dk_{f }}+\mathrm{Dk_{ \textrm {d } }}
\end{aligned}
$$


UNCLASSIF IED

I B-15 KINETICS

1. $\frac{\mathrm{d}}{\mathrm{dt}} \mathrm{s}_{\mathrm{N}}=660.38\left(\mathrm{Dk}_{\mathrm{t}}-1\right) \mathrm{s}_{\mathrm{N}}+0.0276 \mathrm{CI}_{1}+0.235 \mathrm{CI}_{2}+1.65 \mathrm{CI}_{3}$

2. $\frac{\mathrm{d}}{\mathrm{dt}} \mathrm{CI} \mathrm{I}_{1}=180.99 \mathrm{~S}_{\mathrm{N}}-0.0276 \mathrm{CI}_{1}$

3. $\frac{\mathrm{d}}{\mathrm{dt}} \mathrm{CI}_{2}=369.08 \mathrm{~s}_{\mathrm{N}}-0.235 \mathrm{CI}_{2}$

4. $\frac{\mathrm{d}}{\mathrm{dt}} \mathrm{CI}_{3}=110.31 \mathrm{~s}_{\mathrm{N}}-1.65 \mathrm{CI}_{3}$

5. $\frac{\mathrm{d}}{\mathrm{dt}} \mathrm{Q}_{1}=0.000117 \mathrm{~s}_{\mathrm{N}}-0.00322 \mathrm{Q}_{1}$

6. $\frac{d}{d t} Q_{2}=0.000757 S_{N}-0.05525 Q_{2}$

7. $\frac{d}{d t} Q_{3}=0.003734 S_{N}-0.2707 Q_{3}$

8. $\mathrm{S}_{\mathrm{NQ}}=6.672\left(\mathrm{Q}_{1}+\mathrm{Q}_{2}+\mathrm{Q}_{3}\right)+0.6301 \mathrm{~s}_{\mathrm{N}}$

9. $S=S_{N}+Q_{1}+Q_{2}+Q_{3}$
Page 1 of 1

Revision: 4

Date: $4 / 30 / 70$ 
I C-1 NUCLEAR SUBSYSTEM CONTROLS

1. $\mathrm{T}_{15 \mathrm{D}}=$ Independent Variable

2. $\mathrm{T}_{15 \mathrm{E}}=\mathrm{T}_{15 \mathrm{D}}-\mathrm{T}_{15 \mathrm{M}}$

3. $\frac{{ }_{\mathrm{DM}}}{{ }_{\mathrm{DD}}}=\frac{1}{1+.16 \mathrm{~s}+.025 \mathrm{~s}^{2}}$

4. $\frac{{ }_{S} S S V A, B}{{ }_{S S V D}}=\frac{1}{1+.16 S+.025 \mathrm{~S}^{2}}$ 


\section{C-2 PROPELLANT FEEDSYSTEM CONTROLS}

1. $P_{15 E}=P_{15 D}-P_{15}$

2. $\frac{\theta_{S C V D}}{P_{15 E}}=\frac{K(S / 4+1)}{S(S / 100+1)}$

$\mathrm{K}=.005 \mathrm{P}_{15 \mathrm{D}}-1 \quad 270<\mathrm{P}_{15 \mathrm{D}}<450$

$\mathrm{K} \geqq .25$

3. $\frac{{ }^{\theta} \operatorname{SCVA}_{2} B}{{ }_{\operatorname{SCVD}}}=\frac{1}{1.75 \times 10^{-4} s^{2}+1.3 \times 10^{-2} s+1}$

4. $\frac{\theta_{B C V D}}{P_{15 E}}=\frac{-.133(s / 2.5+1)^{2}}{s(s / 300+1)^{2}}$

5. $\frac{\theta_{B C V A, B}}{\theta_{B C V D}}=\frac{1}{1.75 \times 10^{-4} s^{2}+1.3 \times 10^{-2} s+1}$ 


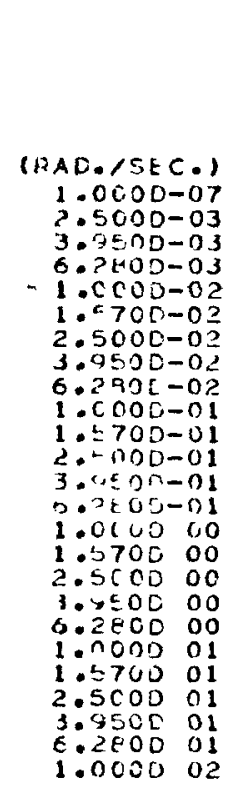

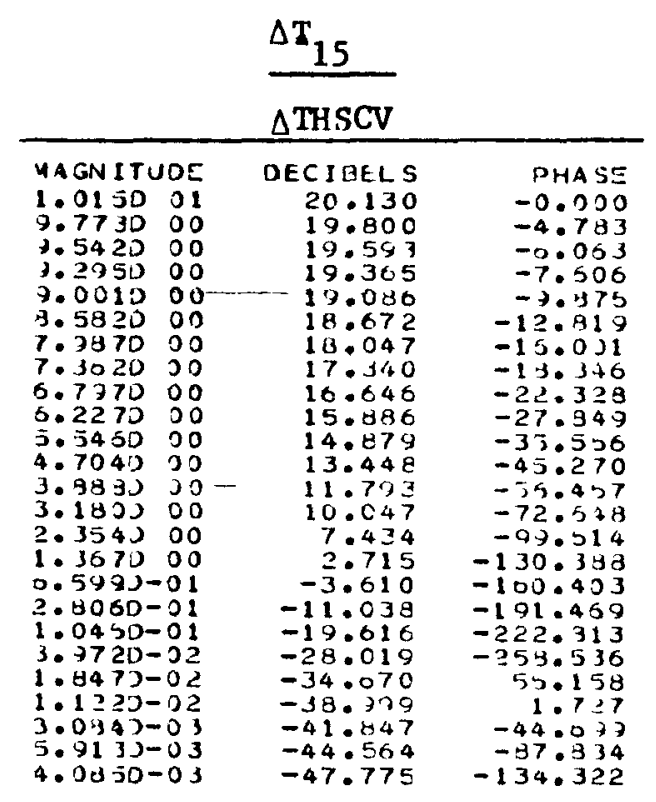

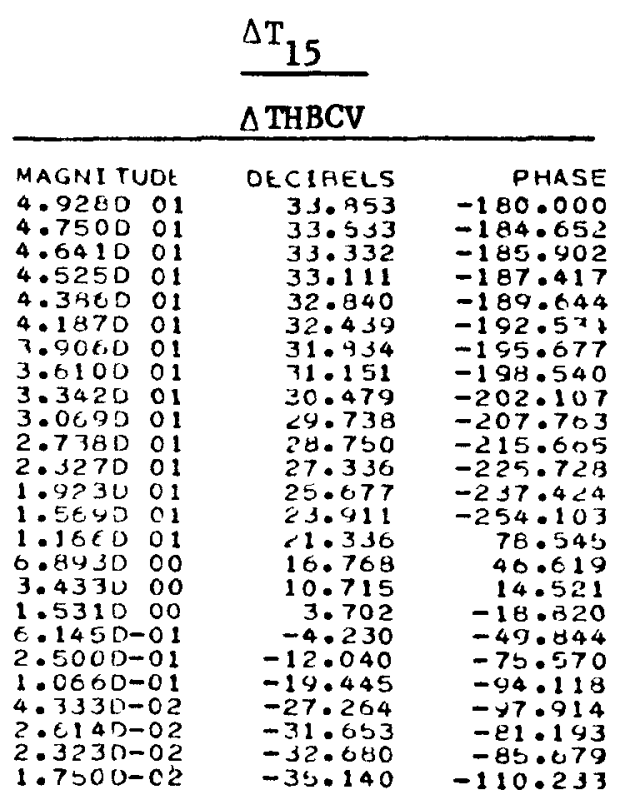

\begin{tabular}{|c|c|c|}
\hline & ${ }^{\Delta T_{15}}$ & \\
\hline & $\triangle \mathrm{THSSV}$ & \\
\hline 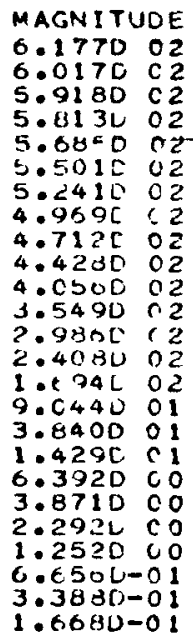 & $\begin{array}{r}\text { DECIBLLS } \\
55.825 \\
55.586 \\
55.444 \\
55.287 \\
-55.093 \\
54.1309 \\
54.3144 \\
53.925 \\
-53.464 \\
52.1324 \\
52.160 \\
51.063 \\
-49.500 \\
47.632 \\
44.578 \\
19.127 \\
-31.087 \\
23.103 \\
16.113 \\
11.757 \\
-7.203 \\
1.954 \\
-3.516 \\
-99.400 \\
-15.558\end{array}$ & 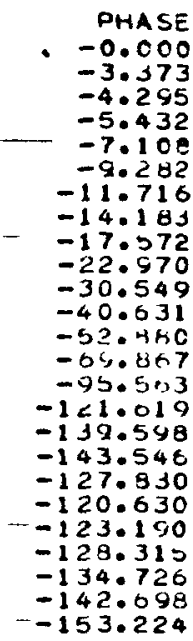 \\
\hline
\end{tabular}

\begin{tabular}{|c|c|c|c|c|c|c|}
\hline & & $\Delta \mathrm{P}_{15}$ & & & $\Delta \mathrm{P}_{15}$ & \\
\hline & & $\triangle \mathrm{THSCV}$ & & & $\triangle \mathrm{THBCV}$ & \\
\hline & & DECIBELS & & & DECIOELS & \\
\hline $\begin{array}{l}2=0070=63 \\
3: 5+1,0-03 \\
3\end{array}$ & $\begin{array}{l}2.009000 \\
1: 3000 \\
1: 33300\end{array}$ & $\begin{array}{l}0.057 \\
5.575 \\
5.274\end{array}$ & 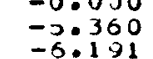 & $\begin{array}{l}9.337000 \\
8.8260 \\
8.5280 \\
8.520\end{array}$ & $\begin{array}{r}19.404 \\
18915 \\
18 \\
1817\end{array}$ & \\
\hline 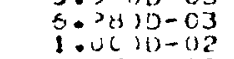 & $\begin{array}{l}1.777000 \\
1: 7250 \\
1\end{array}$ & $\begin{array}{l}4.996 \\
4.736\end{array}$ & $-7: 7$ & $\begin{array}{l}8.2510 \text { oc } \\
8.000 \\
8000\end{array}$ & $\begin{array}{l}18.330 \\
18.06 \mathrm{H}\end{array}$ & \\
\hline 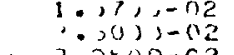 & 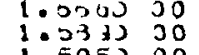 & $\begin{array}{l}4.436 \\
4: 025 \\
4025\end{array}$ & $\begin{array}{rl}-3 & -3049 \\
-10 & 179\end{array}$ & $\begin{array}{ll}0 & 20 \\
0 & 0 \\
0 & 0\end{array}$ & $\begin{array}{l}17.765 \\
17: 3-1 \\
3>1\end{array}$ & \\
\hline 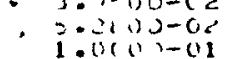 & $\begin{array}{l}1030300 \\
104140 \\
1030400\end{array}$ & $\begin{array}{l}3.549 \\
3: 010 \\
3.05 \\
105\end{array}$ & $\begin{array}{l}12: 340 \\
-17: 750\end{array}$ & $\begin{array}{l}0.49000 \\
6: 0200 \\
600\end{array}$ & 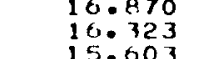 & 90.1 \\
\hline $10-61$ & 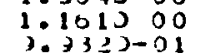 & $\begin{aligned} 1: 300 \\
-0.05\end{aligned}$ & $\begin{array}{l}-200: \\
-21:\end{array}$ & $5: 3$ & 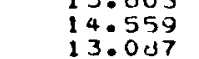 & \\
\hline $\begin{array}{l}13=6 \\
12=0\end{array}$ & $\begin{array}{l}3: 50303731 \\
3=40,3=01\end{array}$ & 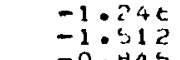 & $\begin{aligned}-17: 13 \\
-12: 52 \\
-12\end{aligned}$ & $\begin{array}{l}7.825000 \\
3.58900 \\
3.000\end{array}$ & $\begin{array}{l}1 \\
11 \\
11: 6933 \\
0.698\end{array}$ & $01 \cdot \frac{5}{2}$ \\
\hline & 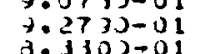 & 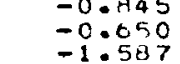 & $\begin{array}{l}-14: 41 \\
-23 \\
-44: 45\end{array}$ & $\begin{array}{l}3.8560 \\
4.0450 \\
3: 8560\end{array}$ & 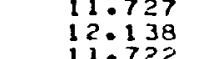 & $2421: 0$ \\
\hline $\begin{array}{l}\text { co } \\
\text { oc }\end{array}$ & $\begin{array}{l}5: 3390-01 \\
3027,-01\end{array}$ & $-4: 30$ & $\begin{array}{ll}-6504 \\
-77: 3\end{array}$ & & & \\
\hline & 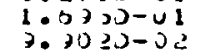 & $\begin{array}{l}-15: 419 \\
20: 018 \\
20.03\end{array}$ & $\begin{array}{l}-73.146 \\
-50: 317\end{array}$ & $\begin{array}{l}1: 40000 \\
1: 110000\end{array}$ & $\begin{array}{l}3.751 \\
1.4413\end{array}$ & \\
\hline & & & $120:$ & $\begin{array}{l}9.497 \\
7: 4525\end{array}$ & $\begin{array}{l}=0.0511 \\
=2.586 \\
-283\end{array}$ & $-c$ \\
\hline & & 4.53 & $\begin{array}{l}16: 136 \\
23: 157\end{array}$ & $\begin{array}{l}5.5450-c 1 \\
3.8740-01\end{array}$ & $\begin{array}{l}-5.1233 \\
-8.236\end{array}$ & 57 \\
\hline
\end{tabular}

\begin{tabular}{|c|c|c|}
\hline \multicolumn{3}{|c|}{$\stackrel{\Delta P_{15}}{ }$} \\
\hline & $\triangle \mathrm{THSSV}$ & \\
\hline 8 & $\begin{array}{c}D E C 1 B t L L S \\
37.244\end{array}$ & \\
\hline 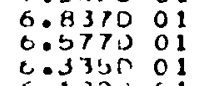 & $\begin{array}{l}36 \\
36 \\
35\end{array}$ & \\
\hline 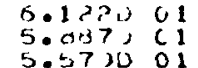 & $\begin{aligned} 35.738 \\
350 \\
34\end{aligned}$ & \\
\hline 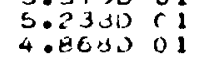 & $34: 543$ & \\
\hline $\begin{array}{l}3940 \\
: 7410\end{array}$ & $\begin{array}{l}31: 867 \\
31: 458\end{array}$ & -40.6 \\
\hline 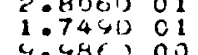 & 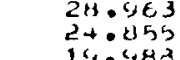 & $\begin{array}{l}-51.9 \\
-513.5 \\
\end{array}$ \\
\hline 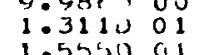 & 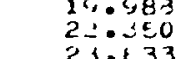 & ci. \\
\hline $\begin{aligned} 4120 \\
64010 \\
600\end{aligned}$ & 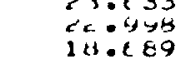 & $\begin{array}{l}480 \\
800 \\
1,50\end{array}$ \\
\hline $\begin{array}{l}1714 \\
0411\end{array}$ & 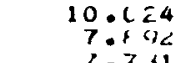 & $\begin{array}{l}113 \\
41\end{array}$ \\
\hline & 1.75 & \\
\hline . $2 \mathrm{BSCL}-01$ & 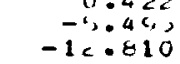 & \\
\hline
\end{tabular}

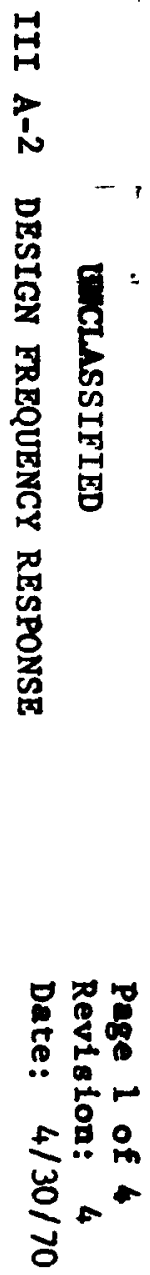

In each case above both of the 2 parallel valves are oscillated.

All actuators and controllers and transducers are deleted. 


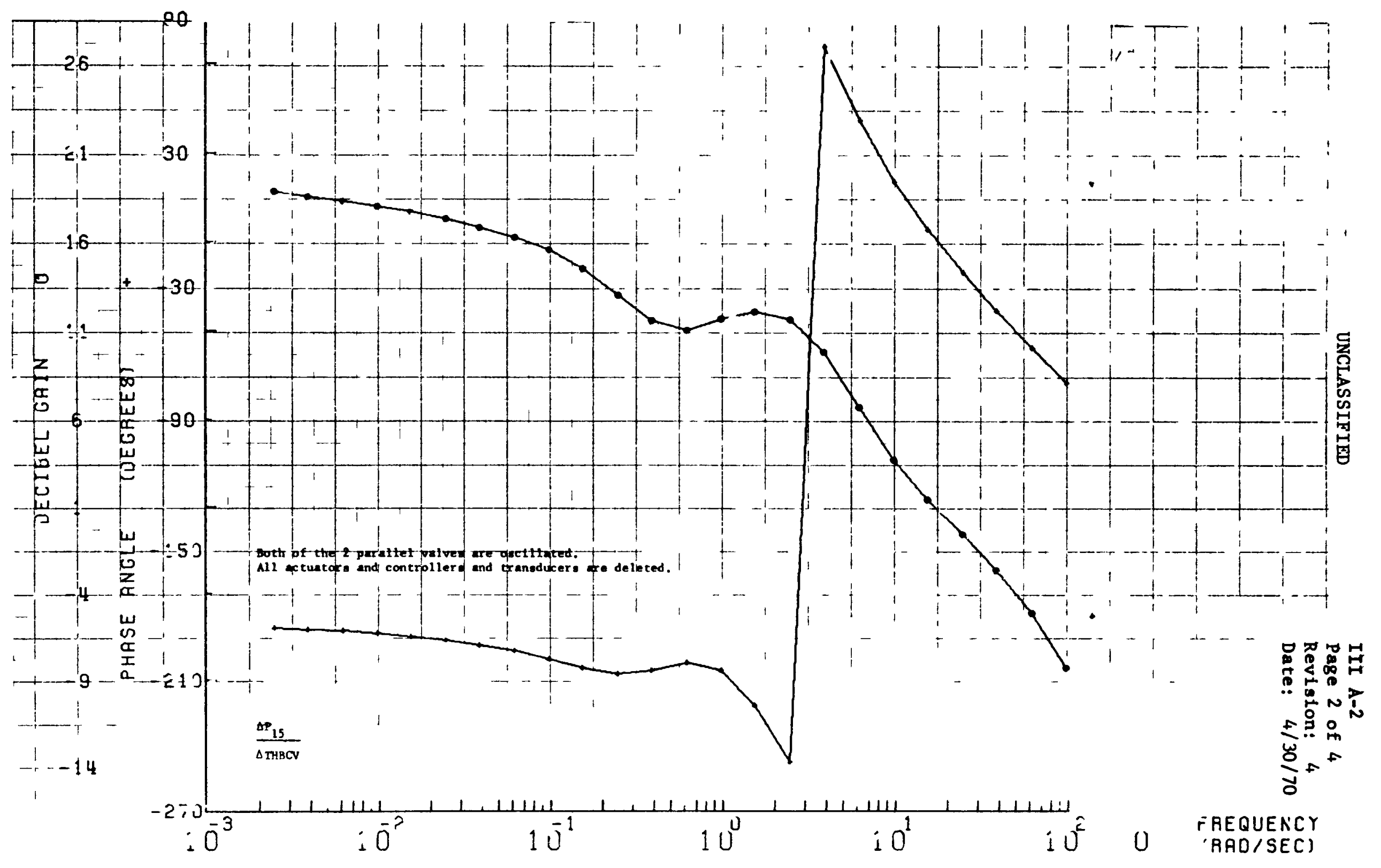




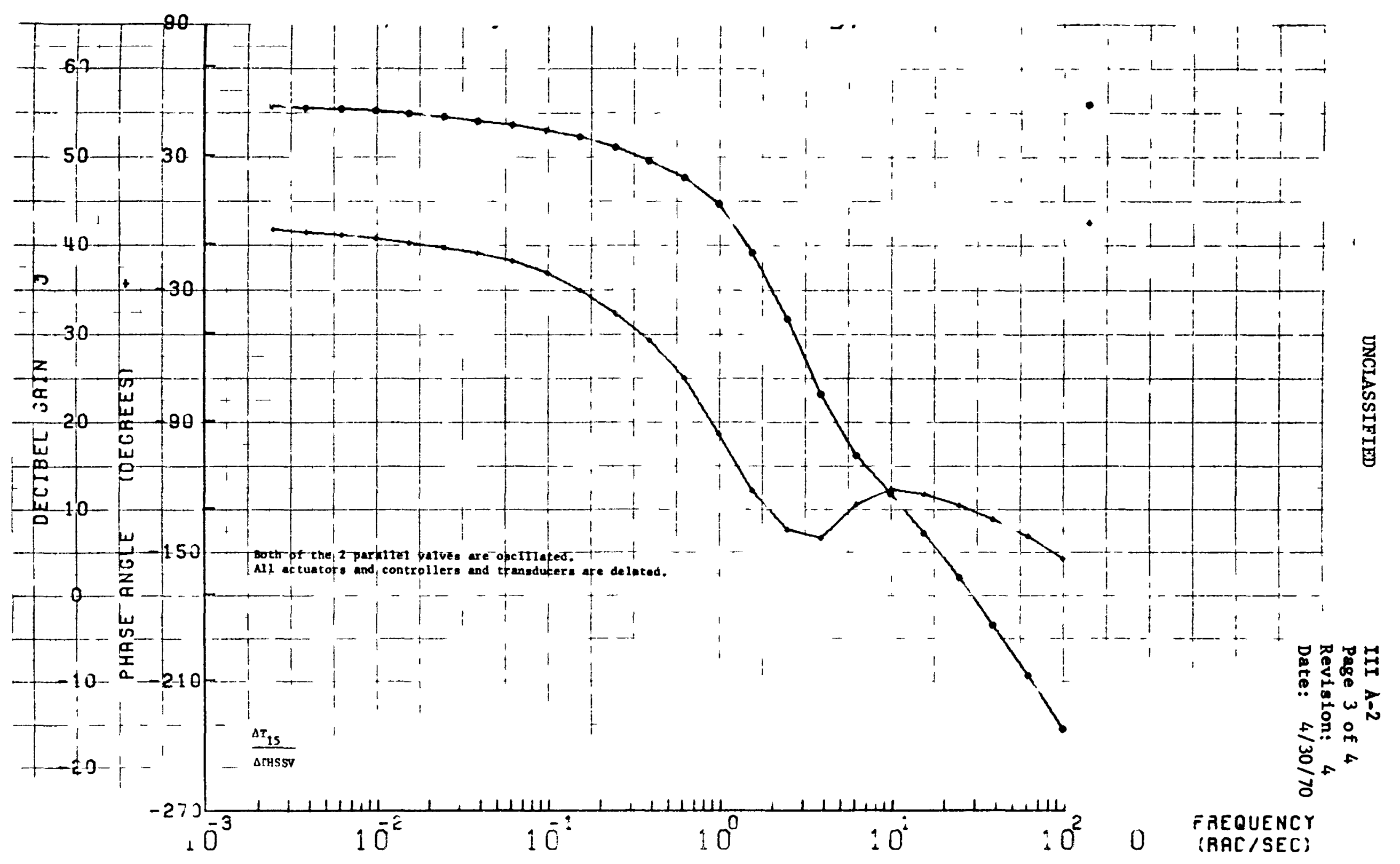




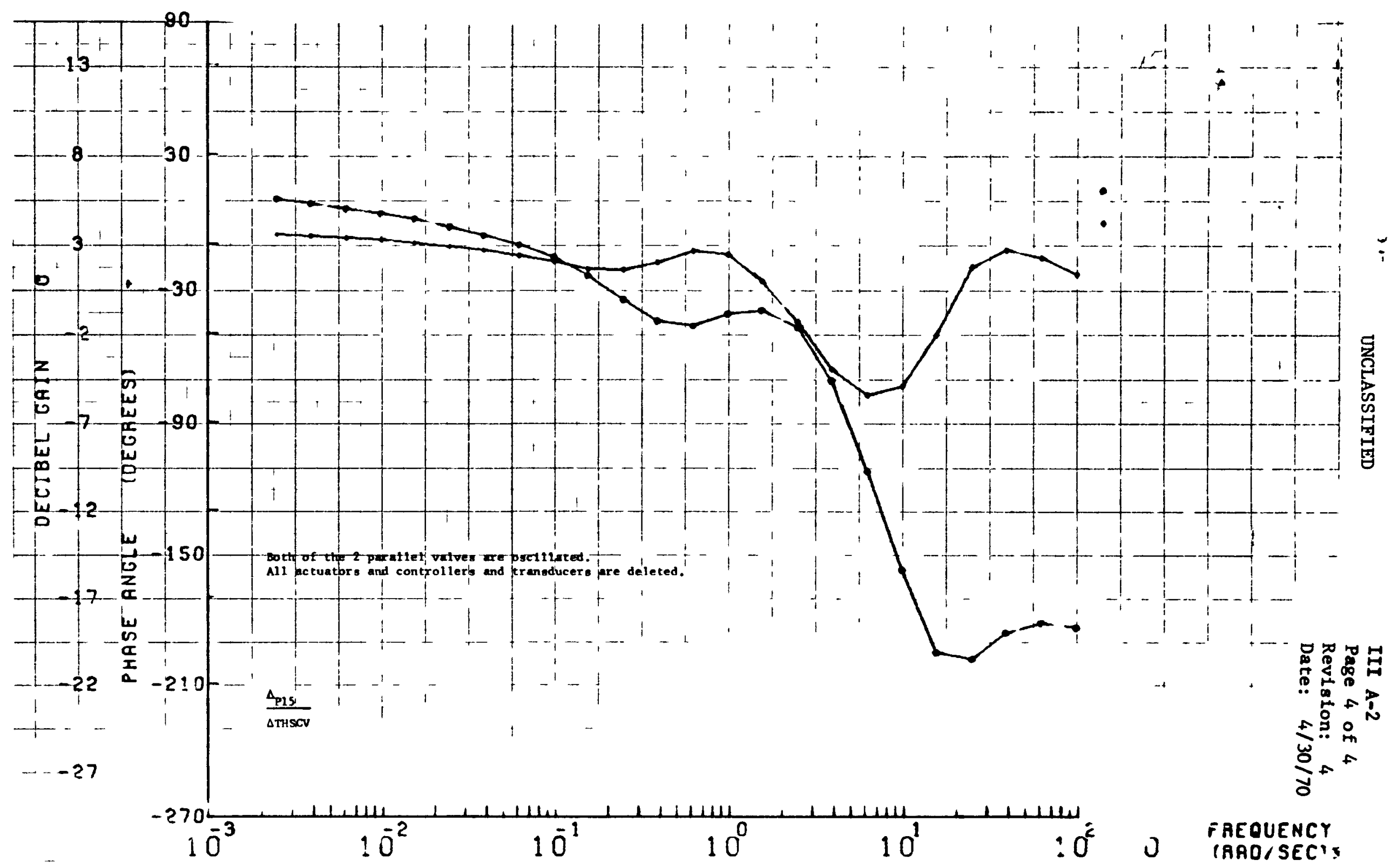




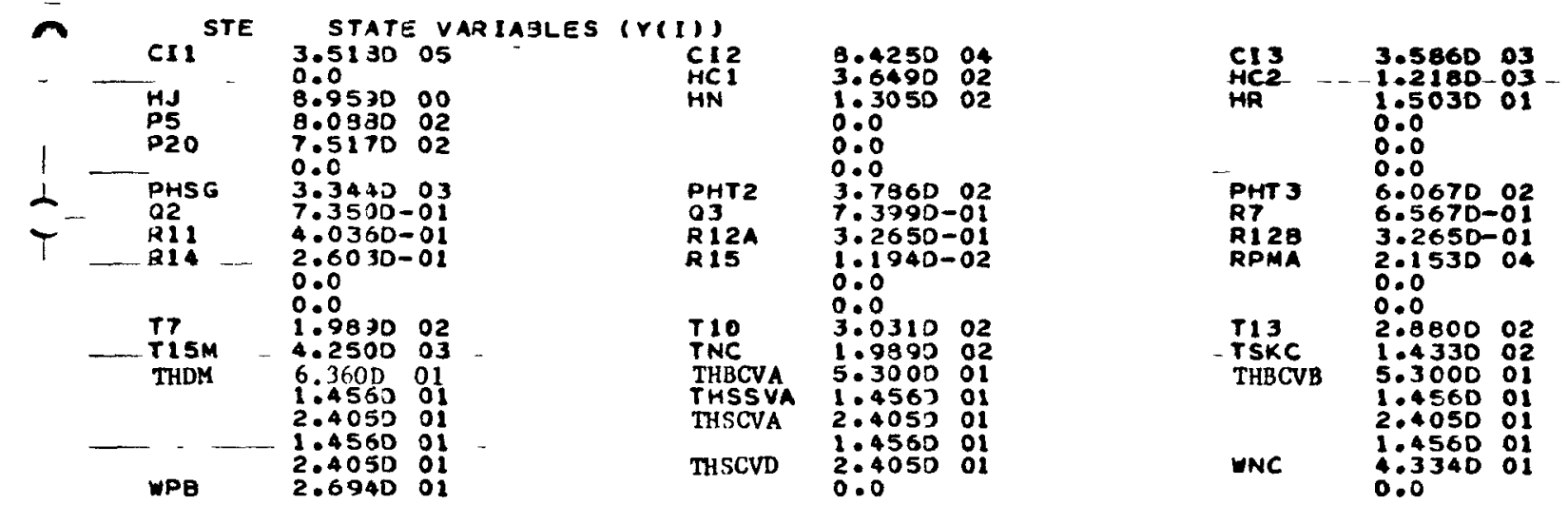

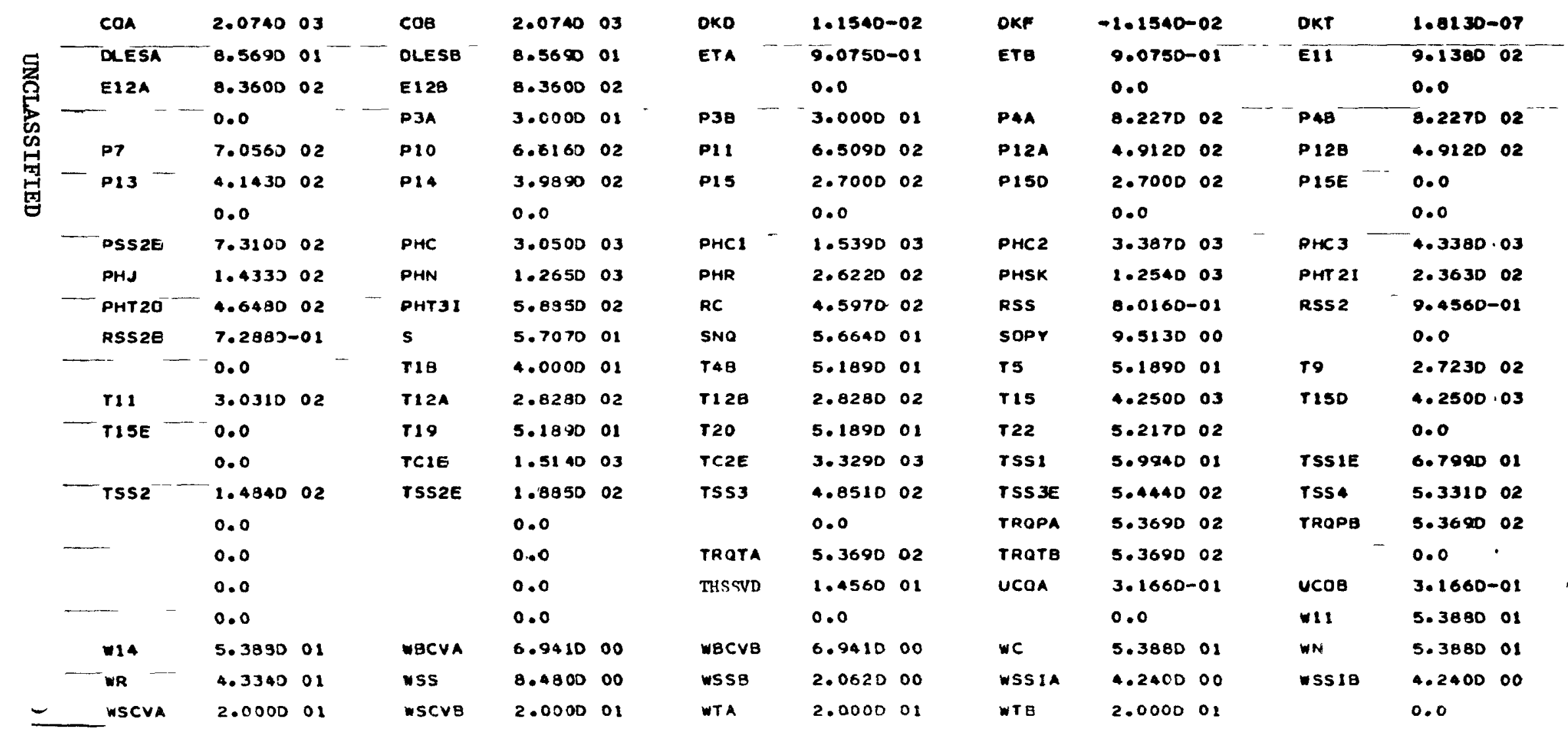
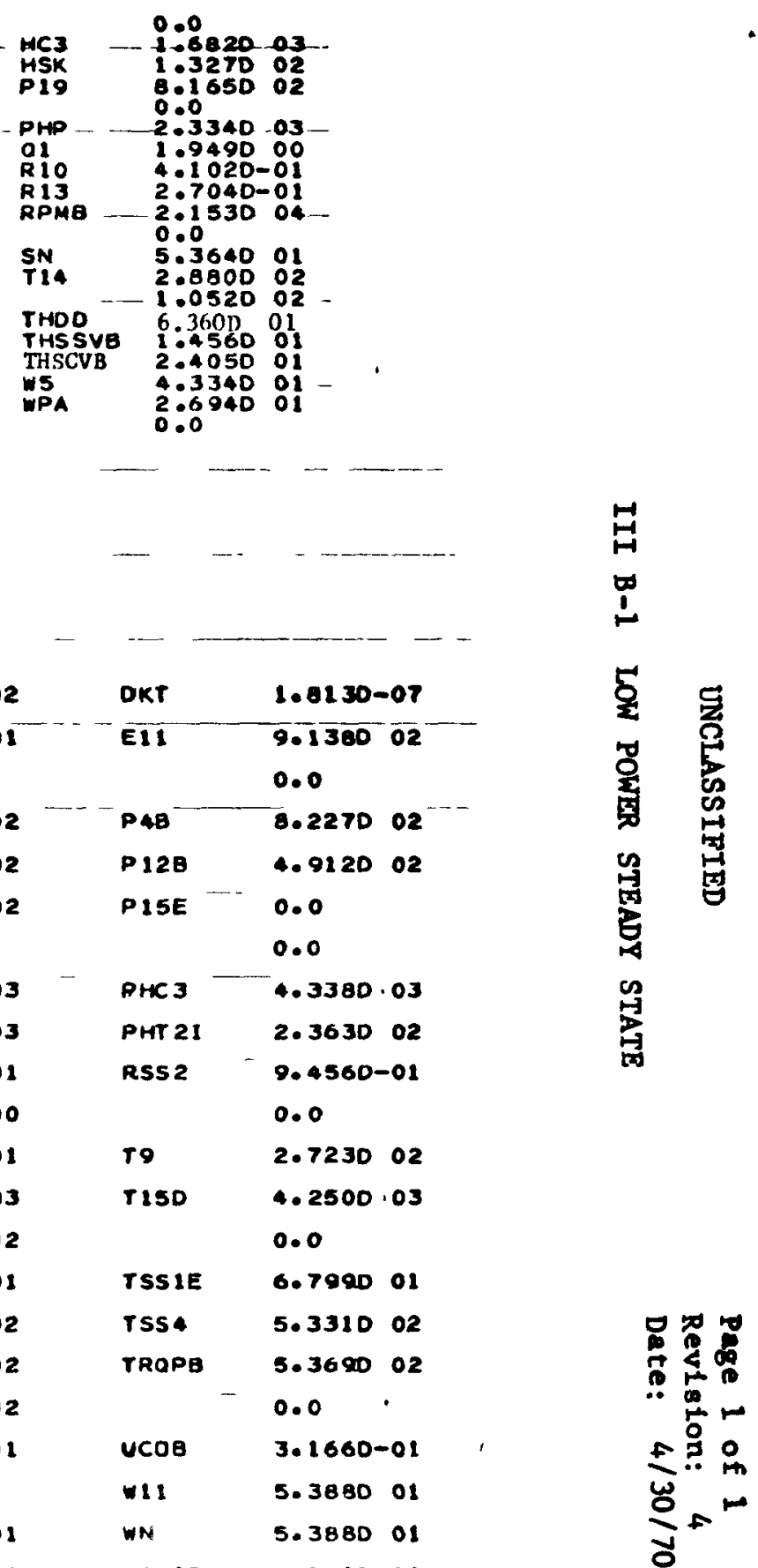


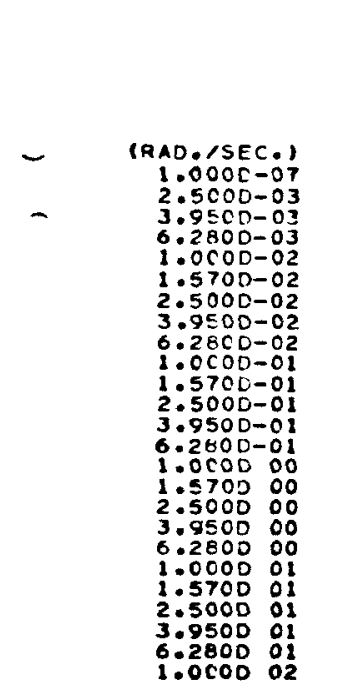

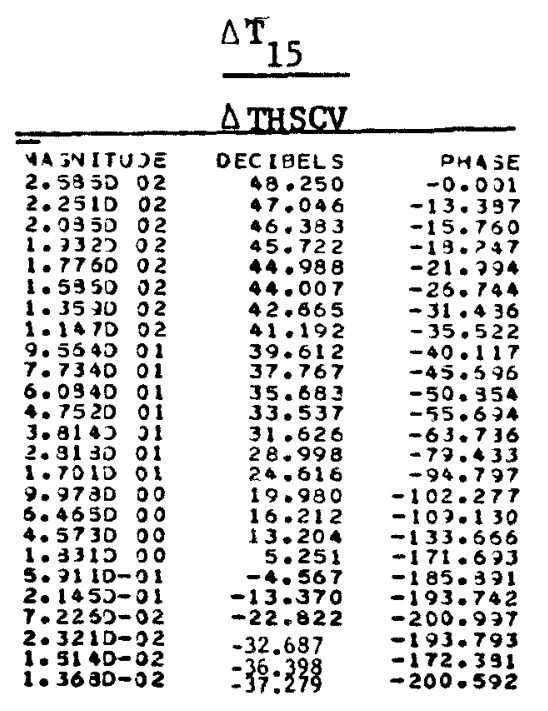

\begin{tabular}{|c|c|c|}
\hline & $\Delta \mathrm{T}_{15}$ & \\
\hline & $\triangle \mathrm{THBCV}$ & \\
\hline 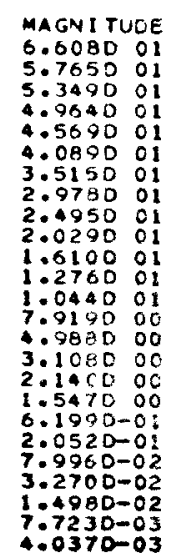 & 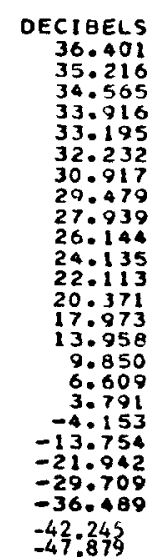 & 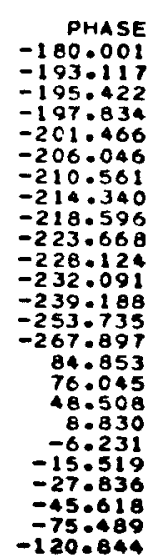 \\
\hline
\end{tabular}

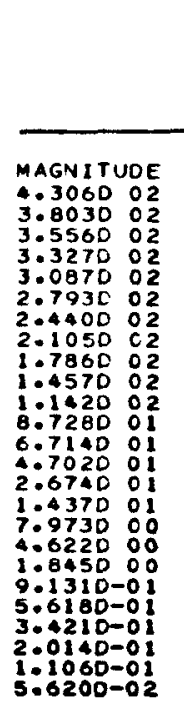

$\Delta \mathrm{T}_{15}$

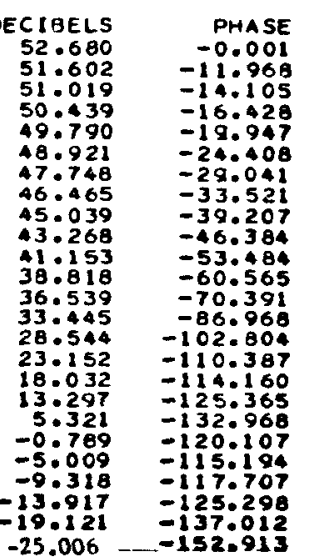

塄

\begin{tabular}{|c|c|c|}
\hline \multicolumn{3}{|c|}{$\Delta \mathrm{P}_{15}$} \\
\hline & $\triangle \mathrm{THSCV}$ & \\
\hline 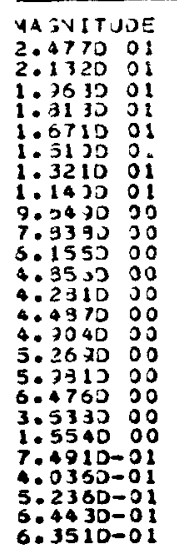 & 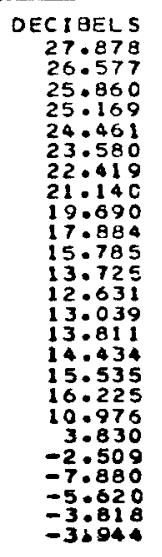 & 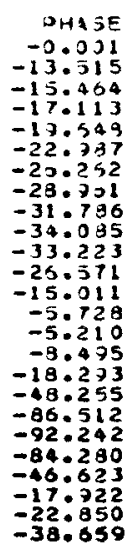 \\
\hline
\end{tabular}

\begin{tabular}{|c|c|c|}
\hline \multicolumn{3}{|c|}{$\Delta \mathrm{P}_{15}$} \\
\hline & $\triangle \mathrm{THBCV}$ & \\
\hline 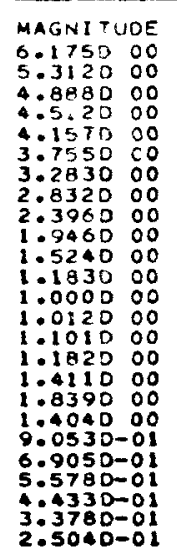 & 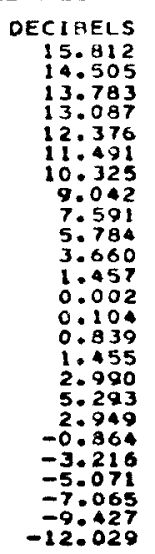 & 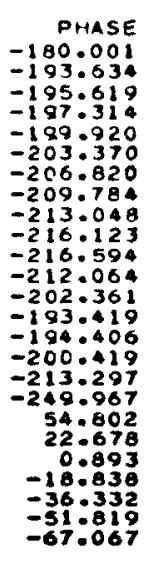 \\
\hline
\end{tabular}

\begin{tabular}{|c|c|c|}
\hline \multicolumn{2}{|c|}{$\Delta \mathrm{P}_{15}$} & \\
\hline & $\triangle \mathrm{THSSV}$ & \\
\hline 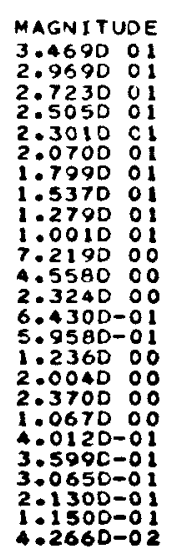 & 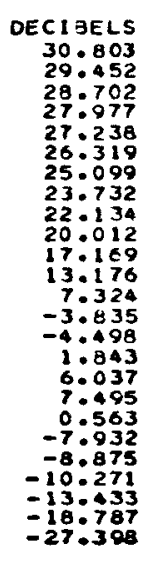 & \\
\hline
\end{tabular}

In each case above both of the 2 parallel valves are oscillated. All actuators and controllers and transducers are deleted. 


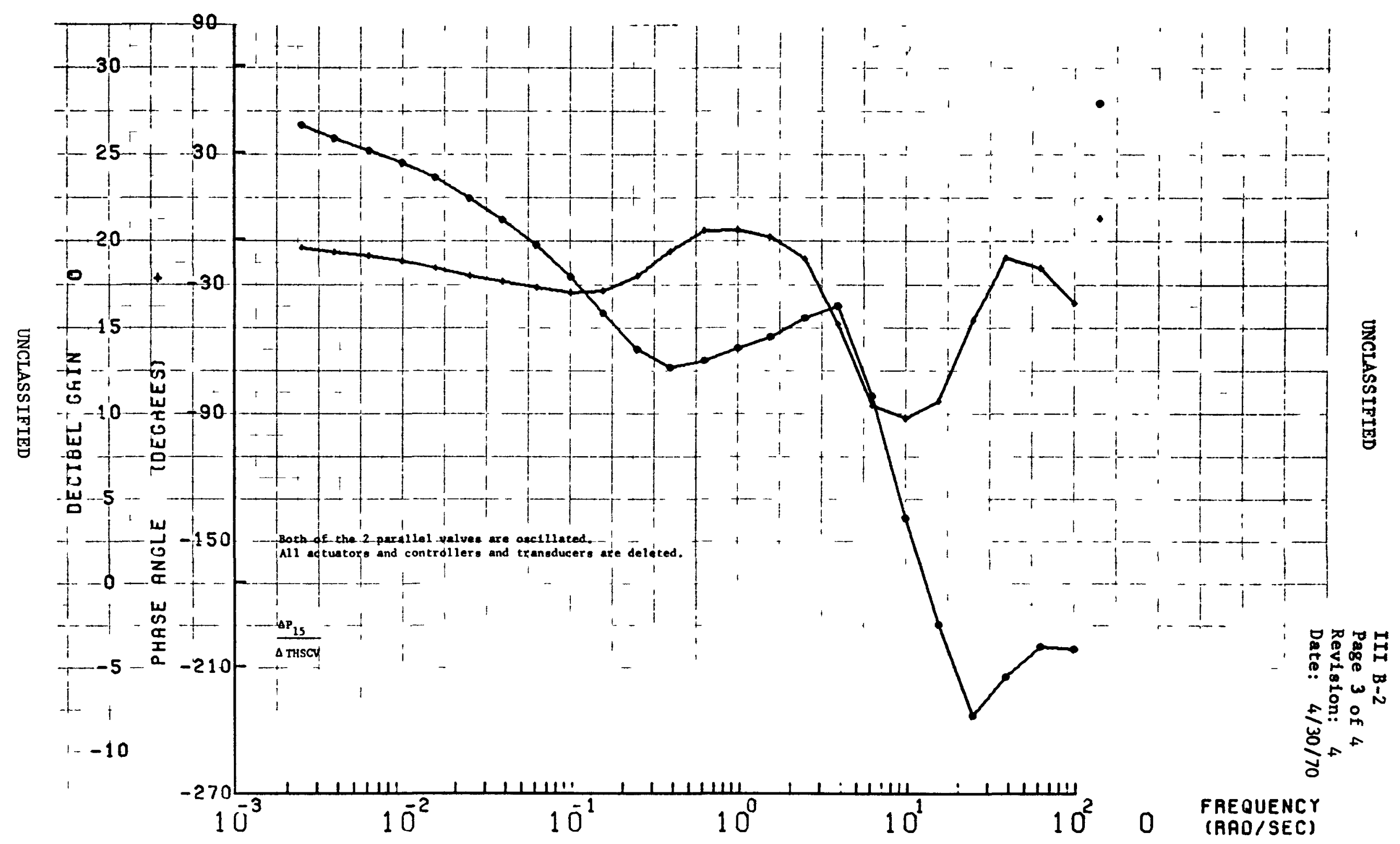


IV NOMENCLATURE

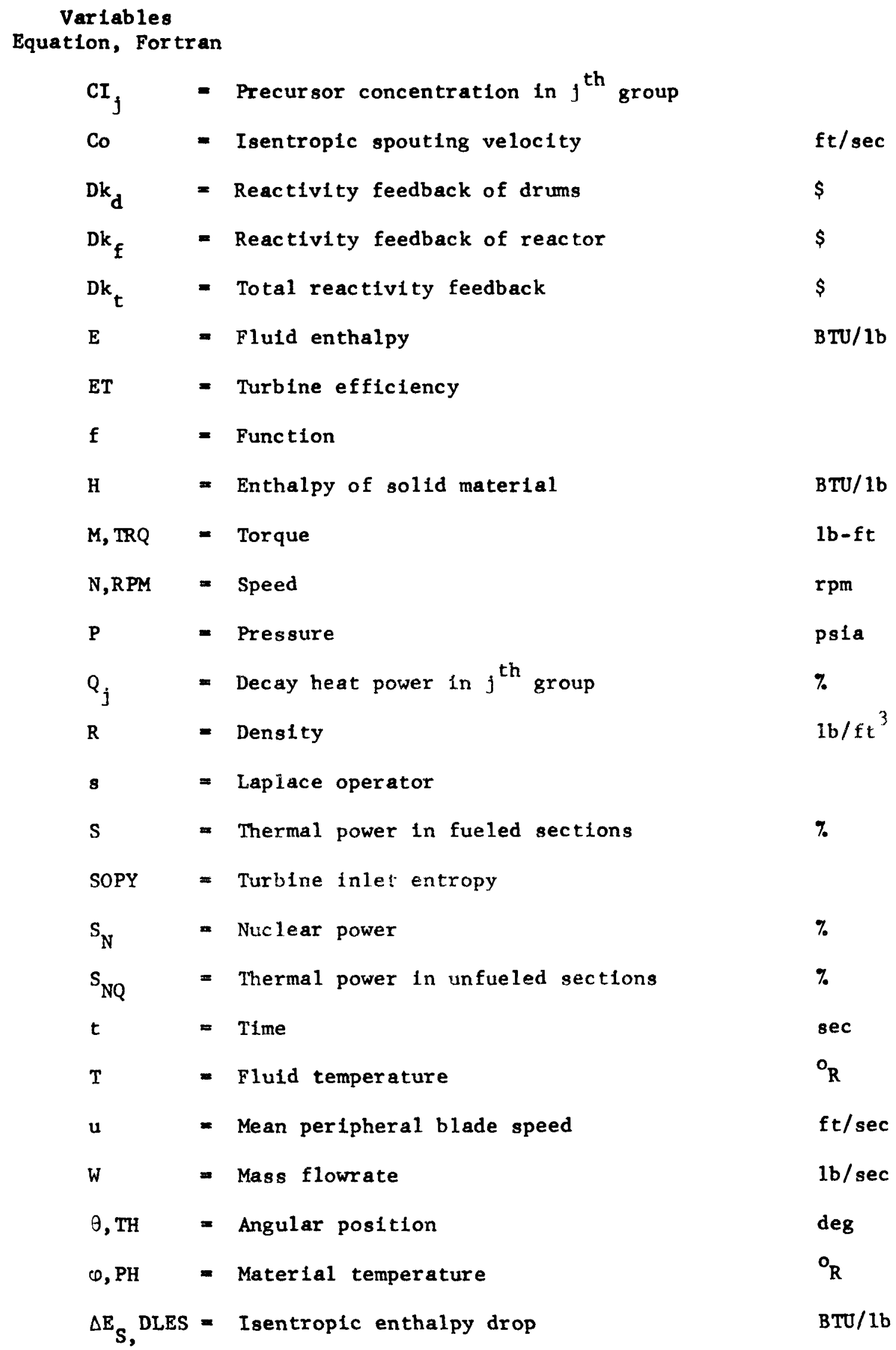


Station

$\begin{array}{ll}3 & =\text { Pump inlet } \\ 4 & =\text { Pump discharge } \\ 5 & =\text { Nozzle inlet } \\ 7 & =\text { Reflector inlet plenum } \\ 9 & =\text { Reflector discharge } \\ 10 & =\text { Peripheral shield exit plenum } \\ 11 & =\text { Bypass and turbine inlet } \\ 12 & =\text { Turbine exit } \\ 13 & =\text { Bypass and series valve exit } \\ 14 & =\text { Shield entrance plenum } \\ 15 & =\text { Nozzle chamber } \\ 19 & =\text { Support structure bypass and control valve inlet } \\ 20 & =\text { Support structure control valve discharge } \\ 22 & =\text { Support system discharge }\end{array}$

Qualifying Subscripts

Equation, Fortran

$\begin{array}{ll}A, B & =\text { Parallel components } \\ B C V & =\text { Bypass control valve } \\ C & =\text { Reactor core, average } \\ C_{1} & =\text { Reactor core, } 1^{\text {th }} \text { section } \\ \text { d } & =\text { Drum } \\ \text { D } & =\text { Demand } \\ \text { e } & =\text { Exit } \\ \text { E } & =\text { Error } \\ j & =\text { Radial inside } \\ J & =\text { Jacket } \\ M & =\text { Measured }\end{array}$


Qualifying Subscripts (continued)

Equation Fortran

$\begin{array}{ll}\mathbf{N} & =\text { Nozzle } \\ \text { NC } & =\text { Nozzle coolant } \\ \text { O } & =\text { Radial outside } \\ \text { P } & =\text { Pyrographite } \\ \text { P } & =\text { Pump } \\ \text { R } & =\text { Reflector } \\ \text { SCV } & =\text { Series control valve } \\ \text { SG } & =\text { Structural graphite } \\ \text { SK } & =\text { Skirt } \\ \text { SKC } & =\text { Skirt coolant } \\ \text { SS } & =\text { Support system average } \\ \text { SSB } & =\text { Support system bypass } \\ \text { SSI } & =\text { Support system inlet } \\ \text { SSj } & =\text { Support system, fth section } \\ \text { ssv } & =\text { Support system control valve } \\ \text { T } & =\text { Turbine } \\ \text { t1 } & =\text { Stainless steel, }{ }^{\text {th }} \text { section }\end{array}$




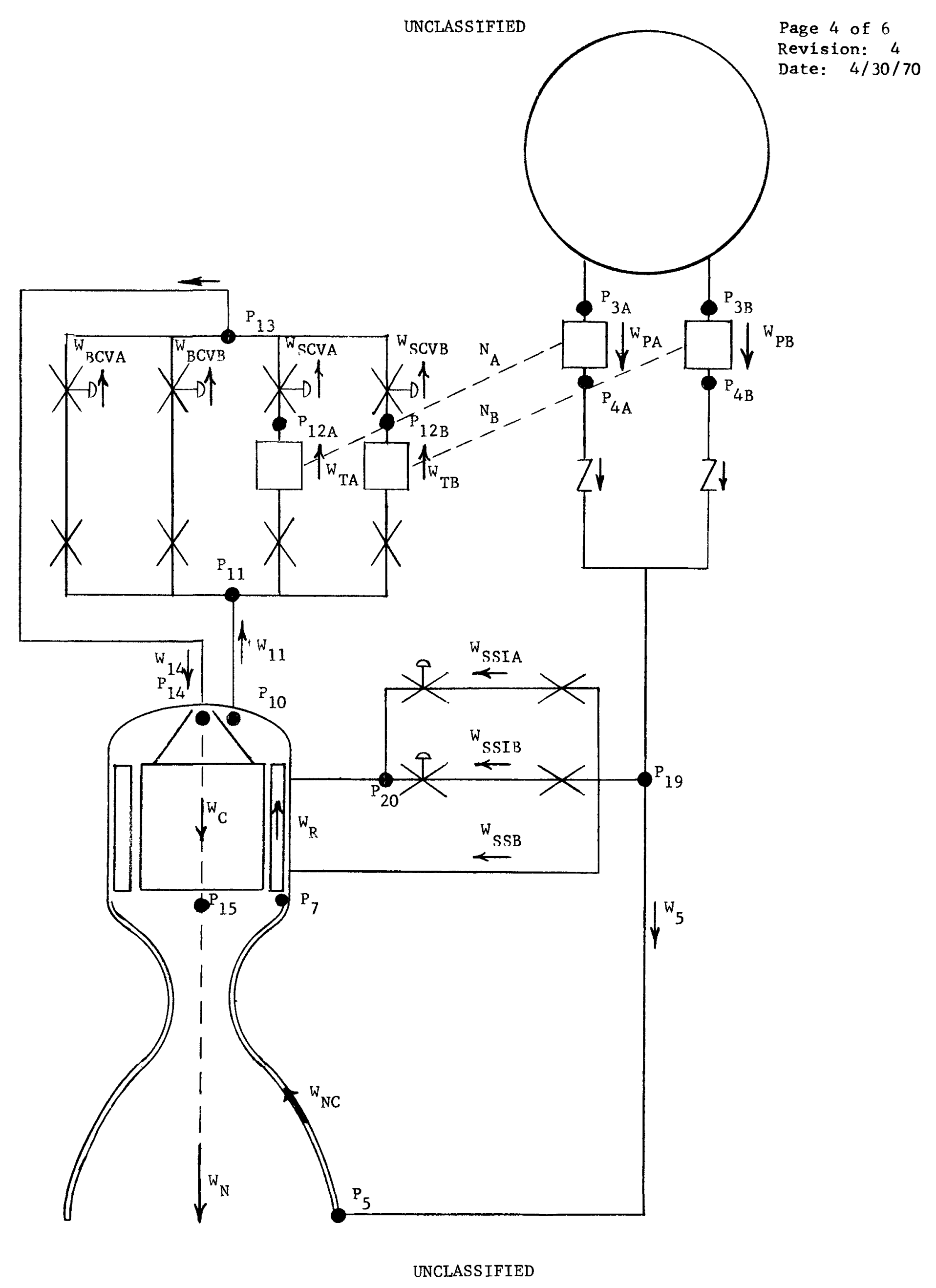


SCHEMATIC DIAGRAM OF E-1 NUCLEAR SUBSYSTEM

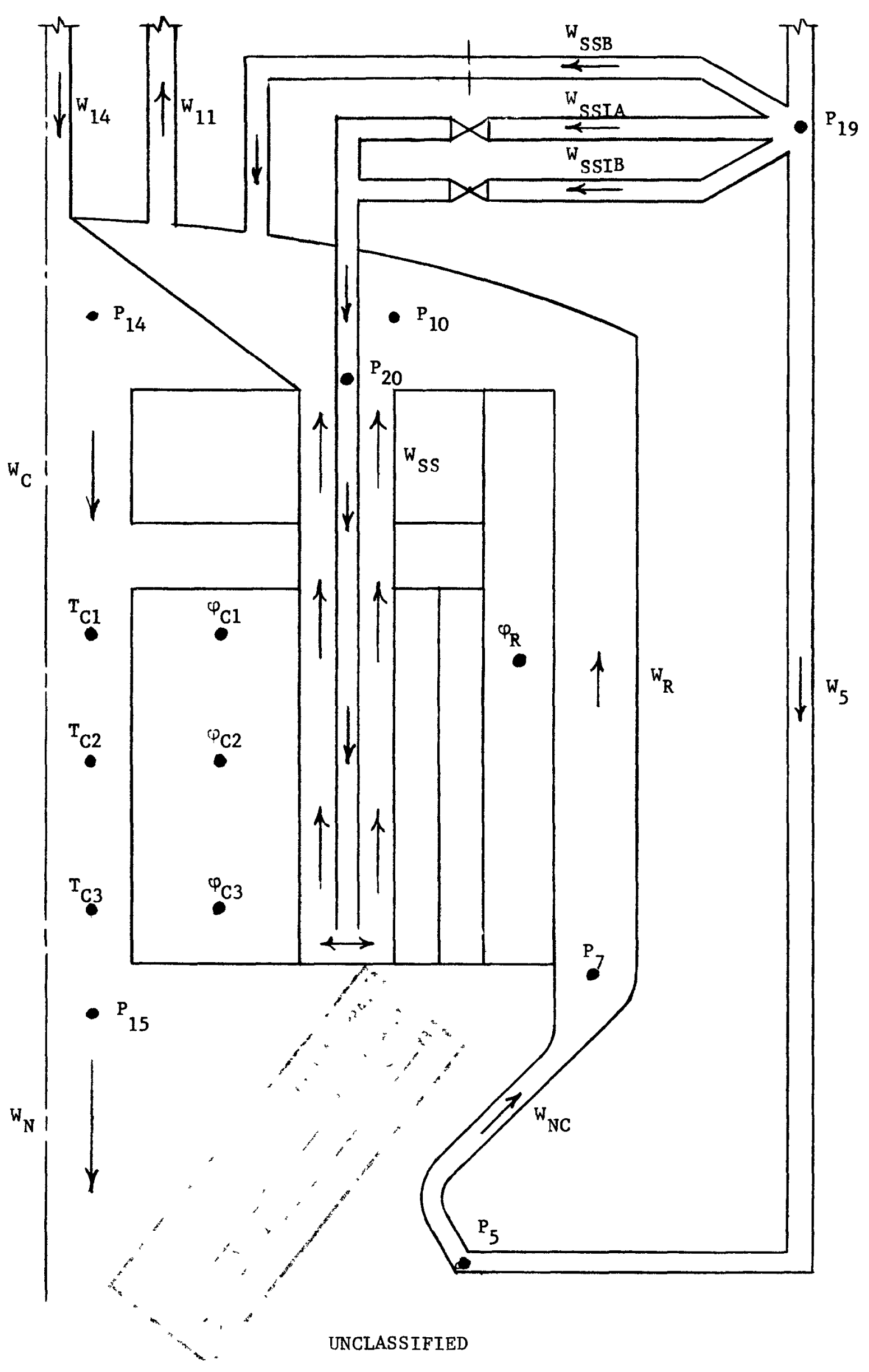




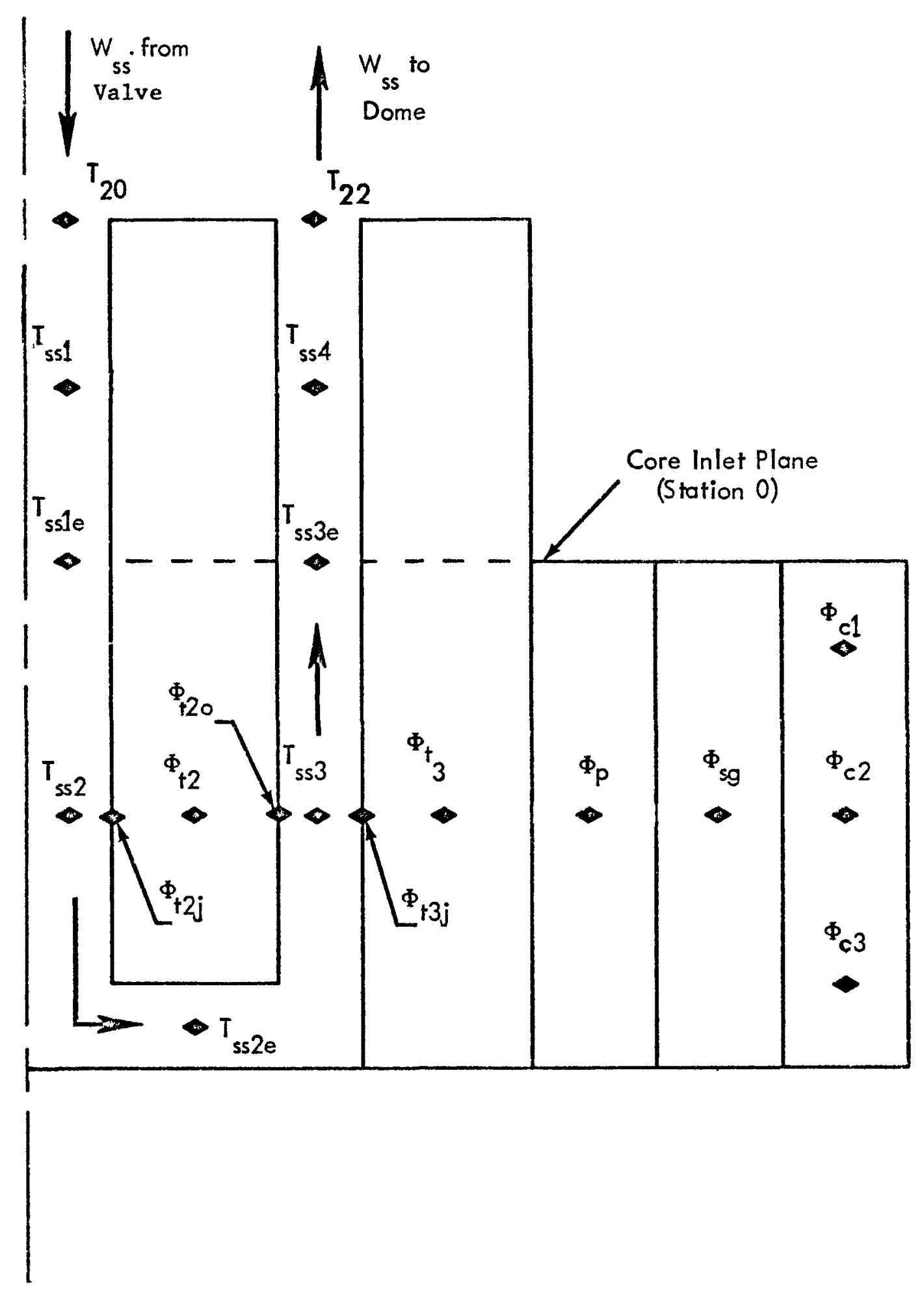

SCHEMATIC DIAGRAM OF SUPPORT SYSTEM (U) 